University of Warwick institutional repository: http://go.warwick.ac.uk/wrap This paper is made available online in accordance with publisher policies. Please scroll down to view the document itself. Please refer to the repository record for this item and our policy information available from the repository home page for further information.

To see the final version of this paper please visit the publisher's website. Access to the published version may require a subscription.

Author(s): P. H. Jefferson, S. A. Hatfield, T. D. Veal, P. D. C. King, C. F. McConville, J. Zúñiga-Pérez, and V. Muñoz-Sanjosé

Article Title: Bandgap and effective mass of epitaxial cadmium oxide Year of publication: 2008

Link to published version: http://dx.doi.org/10.1063/1.2833269

Publisher statement: None 


\title{
Bandgap and effective mass of epitaxial cadmium oxide
}

\author{
P. H. Jefferson, S. A. Hatfield, T. D. Veal, P. D. C. King, and C. F. McConville ${ }^{\text {a) }}$ \\ Department of Physics, University of Warwick, Coventry, CV4 7AL, United Kingdom \\ J. Zúñiga-Pérez ${ }^{\text {b) }}$ and V. Muñoz-Sanjosé \\ Departamento de Fisica Aplicada y Electromagnetismo, Universitat de València, C/Dr. Moliner 50, \\ 46100 Burjassot, Spain
}

(Received 19 November 2007; accepted 15 December 2007; published online 14 January 2008)

\begin{abstract}
The bandgap and band-edge effective mass of single crystal cadmium oxide, epitaxially grown by metal-organic vapor-phase epitaxy, are determined from infrared reflectivity, ultraviolet/visible absorption, and Hall effect measurements. Analysis and simulation of the optical data, including effects of band nonparabolicity, Moss-Burstein band filling and bandgap renormalization, reveal room temperature bandgap and band-edge effective mass values of $2.16 \pm 0.02 \mathrm{eV}$ and $0.21 \pm 0.01 m_{0}$ respectively. (C) 2008 American Institute of Physics. [DOI: 10.1063/1.2833269]
\end{abstract}

Cadmium oxide $(\mathrm{CdO})$, a semiconductor which crystallizes in the rocksalt structure, is almost entirely transparent in the optical region of the electromagnetic spectrum and has high conductivity. Interest in $\mathrm{CdO}$ and other transparent conducting oxides (TCOs) over the past few years has been prompted by the need for optoelectronic devices operating at short wavelengths ${ }^{1}$ and for thin-film photovoltaics and flat panel displays. ${ }^{2}$

Currently, the accepted and frequently cited value for the direct bandgap of $\mathrm{CdO}$ is $2.28 \mathrm{eV}$ as determined from $100 \mathrm{~K}$ thermoreflectance measurements of single crystal $\mathrm{CdO}$ produced using a vapor transport technique. ${ }^{3}$ The calculations on which this work is based used a parabolic conduction band and an electron effective mass of $0.14 m_{0}$. No justification for the choice of effective mass value was given and though the author concluded that the interpretation of the thermoreflectance spectra is qualitatively correct, it was suggested that the inclusion of the effects of conduction band nonparabolicity may produce better agreement with the data.

In this paper, the room temperature bandgap and the band-edge effective mass of single crystal epitaxially grown $\mathrm{CdO}$ are determined from infrared reflectivity, ultraviolet/ visible optical absorption and Hall effect measurements. The nonparabolicity of the conduction band and the competing effects of Moss-Burstein band-filling and bandgap renormalization are explicitly considered.

Single crystal $\mathrm{CdO}$ samples were grown by metalorganic vapor-phase epitaxy (MOVPE) on $r$-plane sapphire substrates using the growth precursors tertiary butanol and dimethylcadmium. Further details on the growth and structural characterization of these samples can be found elsewhere. ${ }^{4}$ The samples were annealed under ultrahigh vacuum at a temperature of $400{ }^{\circ} \mathrm{C}$ for between 2 and $24 \mathrm{~h}$. As a result of this annealing, the free electron concentrations (mobilites) were reduced (increased) from $\sim 1.8$ $\times 10^{20} \mathrm{~cm}^{-3}\left(51 \mathrm{~cm}^{2} \mathrm{~V}^{-1} \mathrm{~s}^{-1}\right)$, for the as-grown samples, to as low (high) as $4.4 \times 10^{19} \mathrm{~cm}^{-3}\left(113 \mathrm{~cm}^{2} \mathrm{~V}^{-1} \mathrm{~s}^{-1}\right)$ for the postgrowth annealed samples.

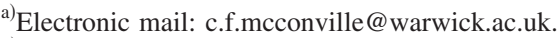

${ }^{b)}$ Present address: CNRS-CRHEA, rue Bernard Grégory, 06560 Valbonne, France.
}

Infrared reflectivity measurements were made using a Perkin Elmer Spectrum GX Fourier transform infrared spectrometer with a $35^{\circ}$ specular reflection with respect to the surface normal. The reflectance was determined from the ratio of the reflection from the $\mathrm{CdO}$ sample and that of a high reflectivity optical mirror. The system employs a cadmium mercury telluride detector giving a working range of 0.05-1.24 eV. Transmission geometry ultraviolet/visible absorption measurements were performed using a Perkin Elmer Lambda 25 spectrometer working between 1.24 and $4.00 \mathrm{eV}$. All measurements from both optical systems were taken at room temperature. Finally, single field Hall effect measurements, also conducted at room temperature, were performed using the standard van der Pauw method.

The infrared reflectivity spectra of three $\mathrm{CdO}$ samples are shown in Fig. 1. The spectra were simulated using an expression describing the propagation and reflection of electromagnetic radiation from a two-layer stratified medium derived from the Fresnel equations. ${ }^{5}$ This expression is dependent on the complex refractive indices $(\tilde{n})$ of the materials which are described in terms of the two-oscillator dielectric model of the complex permittivity $(\widetilde{\boldsymbol{\epsilon}})$ where

$$
\tilde{\epsilon}=\widetilde{n}^{2}=\epsilon(\infty)+\frac{[\epsilon(0)-\epsilon(\infty)] \omega_{T}^{2}}{\omega_{T}^{2}-\omega-i \omega \gamma}-\frac{\epsilon(\infty) \omega_{p}^{2}}{\omega(\omega+i / \tau)} .
$$

Here, $\omega$ is the frequency of the incident radiation, $\epsilon(0)$ and $\epsilon(\infty)$ are the static and high-frequency dielectric constants, $\omega_{T}$ and $\omega_{p}$ are the TO-phonon and plasma frequencies, and $\gamma$ and $\tau$ are the phonon damping and free-carrier scattering time.

Simulation of the reflectivity spectra enables the determination of the plasma frequency and the epilayer thickness. The sample thicknesses obtain from fitting the reflectivity spectra were in the range of $0.48-0.65 \mu \mathrm{m}$, as a result, the samples were treated as fully relaxed as the calculated thicknesses were greater than the $\mathrm{CdO} /$ sapphire critical thickness.

The plasma frequency plotted as a function of carrier concentration for the $\mathrm{CdO}$ samples is shown in Fig. 2 together with calculated values for three different band-edge effective masses. Simulation of the plasma frequency as a function of carrier density was calculated using the following relation 


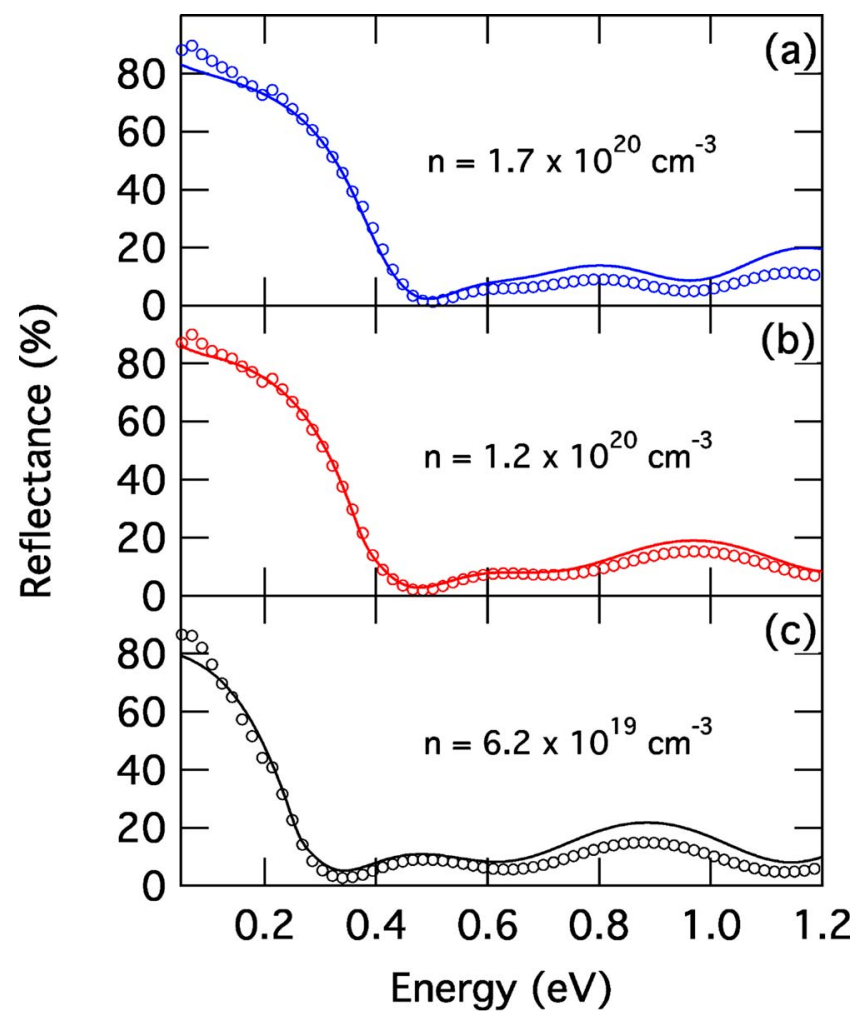

FIG. 1. (Color online) Infrared reflectivity spectra of three CdO samples with carrier concentrations of (a) $1.7 \times 10^{20} \mathrm{~cm}^{-3}$, (b) $1.2 \times 10^{20} \mathrm{~cm}^{-3}$, and (c) $6.2 \times 10^{19} \mathrm{~cm}^{-3}$ (open circles). In each case, the simulation based on the two-oscillator dielectric function is shown (solid lines).

$$
\omega_{p}^{2}=\frac{n e^{2}}{m_{\mathrm{av}}^{*} \epsilon(\infty) \epsilon_{0}},
$$

where $n$ is the free carrier density, $e$ is the electronic charge, $m_{\mathrm{av}}^{*}$ is the density of states integrated effective mass, and $\epsilon_{0}$ the permittivity of free space.

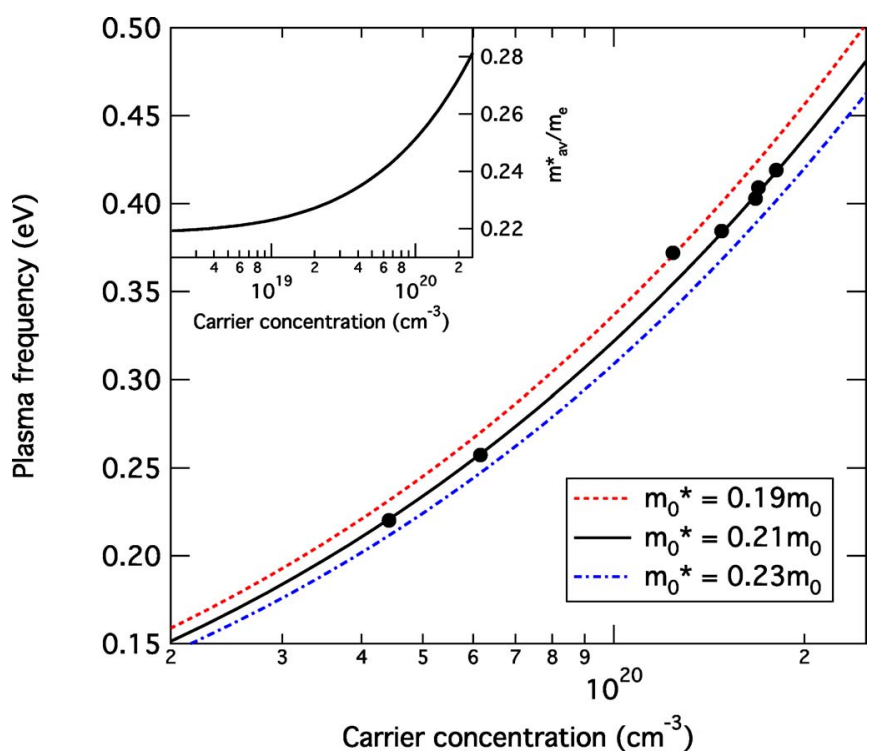

FIG. 2. (Color online) The plasma frequency of the CdO samples determined from infrared reflectivity measurements plotted as a function of carrier concentration (filled circles) together with three simulations of the plasma frequency calculated with a bandgap of $2.16 \mathrm{eV}$ and band-edge effective mass values of $0.19 m_{0}, 0.21 m_{0}$, and $0.23 m_{0}$ (lines). The inset shows the density of states integrated effective mass as a function of carrier concentration. recent density functional theory calculations suggest the
Downloaded 01 Jul 2009 to 137.205.202.8. Redistribution subject to AlP license or copyright; see http://apl.aip.org/apl/copyright.jsp
The value of the effective mass used in Eq. (2) is dependent on the carrier density, since the Fermi level is situated above the conduction band minimum, due to the high electron densities observed in our samples. The effective mass for a given carrier density was calculated by modelling the conduction band dispersion of $\mathrm{CdO}$ using a two-band $\mathbf{k} \cdot \mathbf{p}$ method, and including the effect of bandgap renormalization following Berggren and Sernelius. ${ }^{6}$ Given this band dispersion, the carrier concentration and density of states integrated effective mass for a given Fermi-level $\left(E_{F}\right)$ were determined from

$$
n\left(E_{F}\right)=\int g(E) f\left(E, E_{F}\right) \mathrm{d} E
$$

and

$$
m_{\mathrm{av}}^{*}\left(E_{F}\right)=\frac{1}{n\left(E_{F}\right)} \int m^{*}(E) g(E) f\left(E, E_{F}\right) \mathrm{d} E,
$$

where $g(E)$ is the density of states, $f\left(E, E_{F}\right)$ the Fermi-Dirac distribution, and $m^{*}(E)$ the energy dependent effective mass, calculated from the derivative of the band dispersion.

The plasma frequency was calculated using the bandedge effective mass $\left(m_{0}^{*}\right)$ and the bandgap $\left(E_{g}\right)$ as input parameters. The calculations shown in Fig. 2 have band-edge effective mass values of $0.19 m_{0}, 0.21 m_{0}$, and $0.23 m_{0}$ together with a bandgap value of $2.16 \mathrm{eV}$. The effect of the bandgap value on the plasma frequency simulations was found to be negligible in the range of $1.9-2.3 \mathrm{eV}$.

It can be seen from Fig. 2 that an effective mass of $0.21 m_{0}$ fits the experimentally determined plasma frequencies very well. It should be noted, however, that both the simulations of the infrared reflectivity and the calculated plasma frequency rely on the high-frequency dielectric constant of $\mathrm{CdO}$. A recent paper investigating the effects of the carrier concentration on the dielectric function of various TCOs by spectroscopic ellipsometry suggest that the value of $\epsilon(\infty)$ is linearly reduced with increasing carrier concentration. The reduction in $\epsilon(\infty)$ for $\mathrm{ZnO}$ : Ga was found to be $\sim 5 \%$ for an increase in carrier concentration from 3.2 $\times 10^{19}$ to $7 \times 10^{20} \mathrm{~cm}^{-3}$. Using infrared reflectivity, a unique determination of the high-frequecy dielectric constant and the epilayer thickness is not possible, consequently a previously determined value of 5.3 for $\epsilon(\infty)$ was used. ${ }^{8}$

The ultraviolet/visible $\alpha^{2}$ spectra of three $\mathrm{CdO}$ samples are shown in Fig. 3. The position of the fundamental direct optical transition was obtained from the point of intersection between the linear extrapolations of the indirect absorption and the absorption from direct transitions seen in the vicinity of the $\mathrm{CdO}$ direct bandgap.

The high unintentional $n$-type conductivity observed in $\mathrm{CdO}$ results in a strong dependence of absorption edge with carrier concentration ${ }^{9,10}$ as well as the less pronounced effect of bandgap renormalization resulting from the electronelectron and electron-defect interactions. ${ }^{6}$ As with the calculation of plasma frequency, the Fermi level as a function of carrier concentration is determined from a nonparabolic twoband $\mathbf{k} \cdot \mathbf{p}$ conduction band. Making the assumption that direct transitions take place from a single, nondispersive valence band to the Fermi-level of the nonparabolic conduction band, the position of the fundamental direct absorption feature is obtained. The nondispersive valence band was used as 


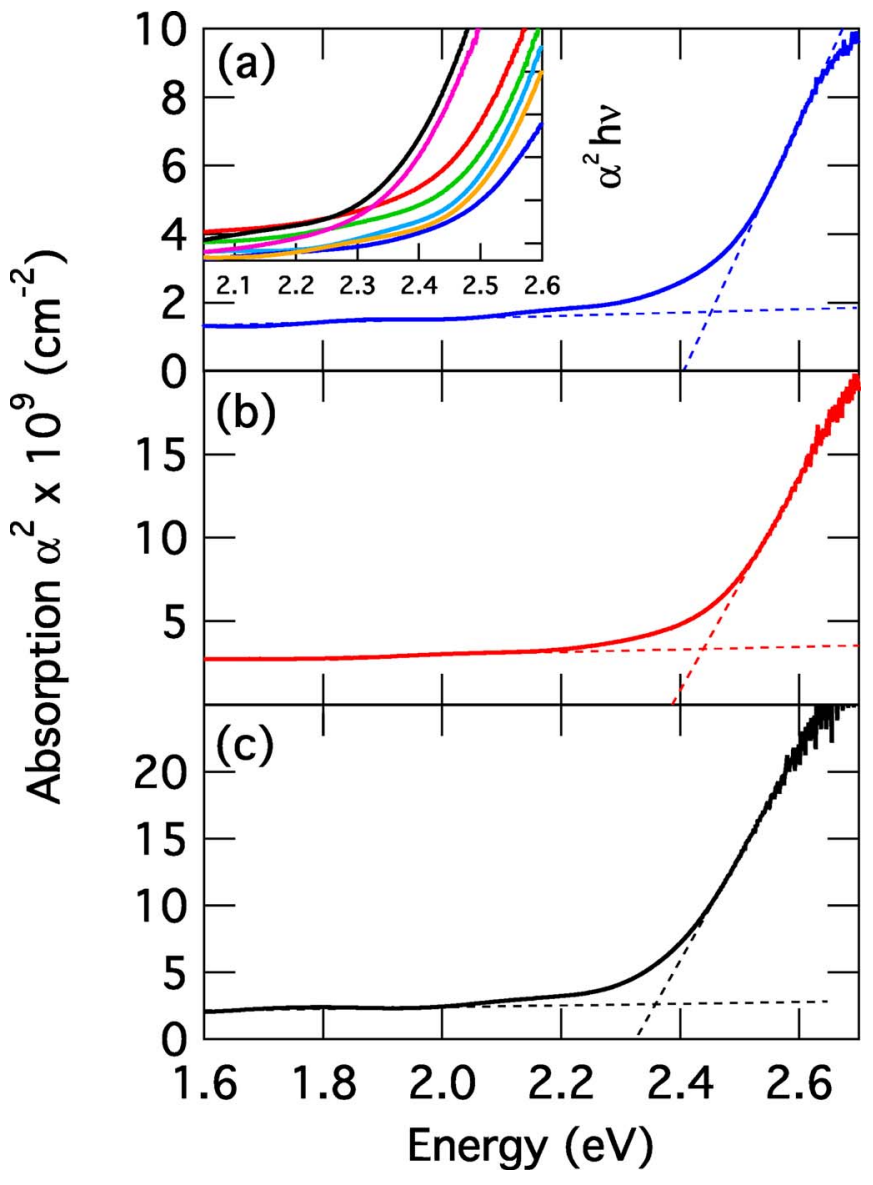

FIG. 3. (Color online) The ultraviolet/visible $\alpha^{2}$ spectra of three CdO samples with carrier concentrations of (a) $1.7 \times 10^{20} \mathrm{~cm}^{-3}$, (b) 1.2 $\times 10^{20} \mathrm{~cm}^{-3}$, and (c) $6.2 \times 10^{19} \mathrm{~cm}^{-3}$. The position of the fundamental optical transition (from valence band to Fermi level) is seen to increase with carrier concentration. This trend is further highlighted in the inset where the $\alpha^{2} \mathrm{~h} \nu$ spectra of all samples considered in this work are shown.

heavy-hole band of rocksalt $\mathrm{CdO}$ changes by less than $50 \mathrm{meV}$ within the region of $k$ space of interest, increasing to maxima at the $L$ point and between the $\Gamma$ and $X$ points. ${ }^{11}$

The calculated absorption edge as a function of carrier concentration is shown in Fig. 4 for bandgap values of 2.14, 2.16 , and $2.18 \mathrm{eV}$ and with the previously determined bandedge effective mass value of $0.21 \mathrm{~m}_{0}$. It can be seen that good agreement between the calculated and experimentally determined values is found within this narrow range of bandgap energies.

In conclusion, infrared reflectivity and ultraviolet/visible optical absorption spectroscopies have been used, together with Hall effect measurements, to determine the bandgap and band-edge effective mass of single-crystal epitaxial cadmium oxide grown by MOVPE on $r$-plane sapphire substrates. The

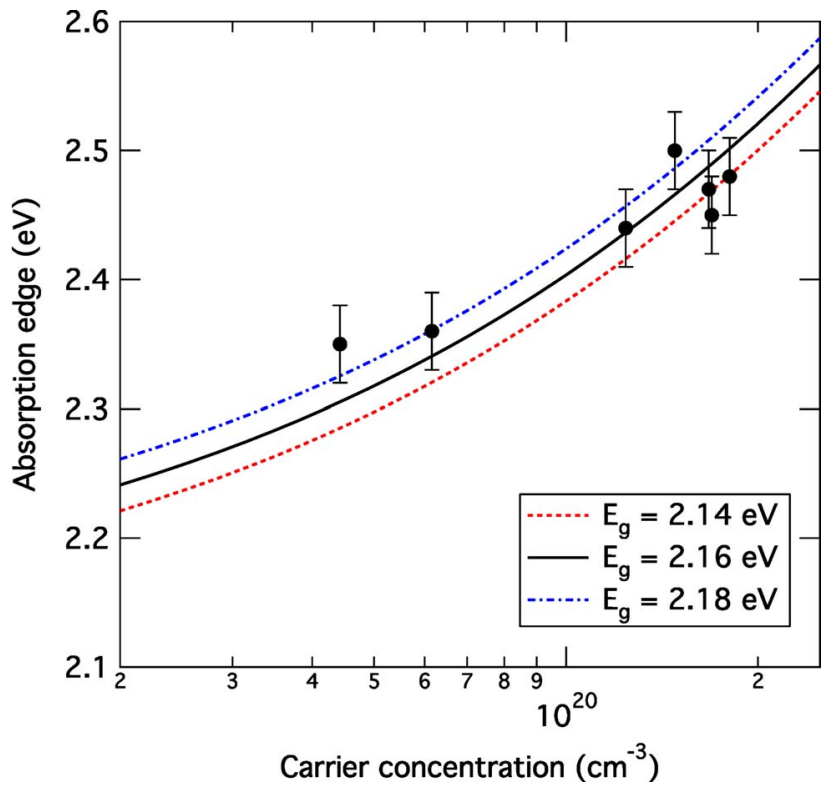

FIG. 4. (Color online) The position of the fundamental optical transition plotted as a function of carrier concentration for the $\mathrm{CdO}$ samples together with simulations calculated using a nonparabolic two-band $\mathbf{k} \cdot \mathbf{p}$ conduction band with a band-edge effective mass of $0.21 m_{0}$ and bandgaps of 2.14, 2.16, and $2.18 \mathrm{eV}$.

band-edge effective mass and bandgap values were found to be $0.21 \pm 0.01 m_{0}$ and $2.16 \pm 0.02 \mathrm{eV}$, respectively. The bandgap value obtained was lower than the value frequently cited as the room-temperature bandgap determined by Koffyberg from thermoreflectance measurements performed at $100 \mathrm{~K}$.

The Engineering and Physical Sciences Research Council, U.K. is acknowledged for financial support under grant number EP/E010210/1.

${ }^{1}$ A. Tsukazaki, Nat. Mater. 4, 42 (2005)

${ }^{2}$ T. J. Coutts, D. L. Young, X. Li, W. P. Mulligan, and X. Wu, J. Vac. Sci. Technol. A 18, 2646 (2000).

${ }^{3}$ F. P. Koffyberg, Phys. Rev. B 13, 4470 (1976).

${ }^{4}$ J. Zúniga-Pérez, C. Munuera, C. Ocal, and V. Munoz-Sanjosé, J. Cryst. Growth 271, 223 (2004).

${ }^{5}$ C. Pickering, J. Phys. C 13, 2959 (1980).

${ }^{6}$ K. F. Berggren and B. E. Sernelius, Phys. Rev. B 24, 1971 (1981).

${ }^{7}$ H. Fujiwara and M. Kondo, Phys. Rev. B 71, 075109 (2005).

${ }^{8} \mathrm{H}$. Finkenrath, in Physics of II-VI and I-VII Compounds, Semi-Magnetic Semiconductors, Landolt-Börnstein: Numerical Data and Functional Relationships in Science and Technology. Group III: Crystal and Solid State Physics, Vol. 17B, edited by O. Madelung, M. Schulz, and H. Weiss (Springer, Berlin, 1982).

${ }^{9}$ T. S. Moss, Proc. Phys. Soc. London, Sect. B 67, 775 (1954).

${ }^{10}$ E. Burstein, Phys. Rev. 93, 632 (1954).

${ }^{11}$ A. Schleife, F. Fuchs, J. Furthmüller, and F. Bechstedt, Phys. Rev. B 73, 245212 (2006). 